\title{
Glycemic targets for elderly patients with diabetes
}

\author{
Japan Diabetes Society (JDS)/Japan Geriatrics Society (JGS) Joint Committee on \\ Improving Care for Elderly Patients with Diabetes $\cdot$ Masakazu Haneda ${ }^{1}$ • \\ Hideki Ito ${ }^{2}$
}

Received: 7 October 2016/Published online: 29 November 2016

(c) The Japan Diabetes Society and the Japan Geriatrics Society 2016 literature, and developed the "Glycemic Targets for Elderly Patients with Diabetes" described in the Table 1.

The Joint Committee's consensus is summarized as follows:

With the accelerated aging of society, the number of elderly patients with diabetes continues to rise in Japan [1]. The elderly have specific health problems that vary widely among individuals. In particular, susceptibility to severe hypoglycemia is a hallmark of elderly diabetes [2, 3]. Severe hypoglycemia not only impairs cognitive function [4] but can also increase the risk of cardiovascular events [5]. Against this background, the Japan Diabetes Society (JDS)/Japan Geriatrics Society (JGS) Joint Committee on Improving Care for Elderly Patients with Diabetes was launched in April 2015. As a first step toward developing "Clinical Practice Guidelines for the Management of Elderly Patients with Diabetes," the Joint Committee launched an in-depth debate on glycemic targets for elderly diabetes.

Over a series of discussions, the Joint Committee drew on the available reports on glycemic targets for the elderly, which included the Japanese Elderly Diabetes Intervention Trial (J-EDIT) study [6], the American Diabetes Association (ADA)/American Geriatrics Society (AGS) Consensus report [7], International Diabetes Federation (IDF)'s Global Guideline [8], and other relevant reports in the pertinent

Members of the Japan Diabetes Society (JDS)/Japan Geriatrics Society (JGS) Joint Committee on Improving Care for Elderly Patients with Diabetes are listed in Appendix.

Masakazu Haneda

jdstokyo@za2.so-net.ne.jp

Asahikawa Medical University, Asahikawa, Hokkaido, Japan

2 Tokyo Metropolitan Geriatric Hospital and Institute of Gerontology, Itabashi, Tokyo, Japan
1. The glycemic target is to be determined for each elderly patient by taking into account the patient's background characteristics and health status [e.g., age, cognitive function, physical function (basic and instrumental activities of daily living, ADL)], comorbidities, risk for severe hypoglycemia, and life expectancy;

2. The lower limit of the glycemic target is to be targeted to ensure safer glycemic control in those likely to be at risk of severe hypoglycemia;

3. The Joint Committee's recommendations allow the glycemic targets to be adjusted to exceed or fall below the glycemic targets suggested in the table to ensure patient-centered care while providing the glycemic targets and their lower limits as a basis for glycemic control in elderly patients with diabetes.

\section{Important precautions}

Be sure to refer to the "Guidelines for Medical Treatment and its Safety in the Elderly" compiled by the JGS for use of antidiabetic agents in the elderly [11, 12]. Every effort should also be made to avoid multi-drug combination therapy in the elderly and to closely monitor these patients for any adverse reaction to drug therapy.

\section{Appendix}

In 2016, the Japan Diabetes Society (JDS)/Japan Geriatrics Society (JGS) Joint Committee on Improving Care for Elderly Patients with Diabetes published one part of the 
Table 1 Glycemic targets (HbA1c values) for elderly patients with diabetes

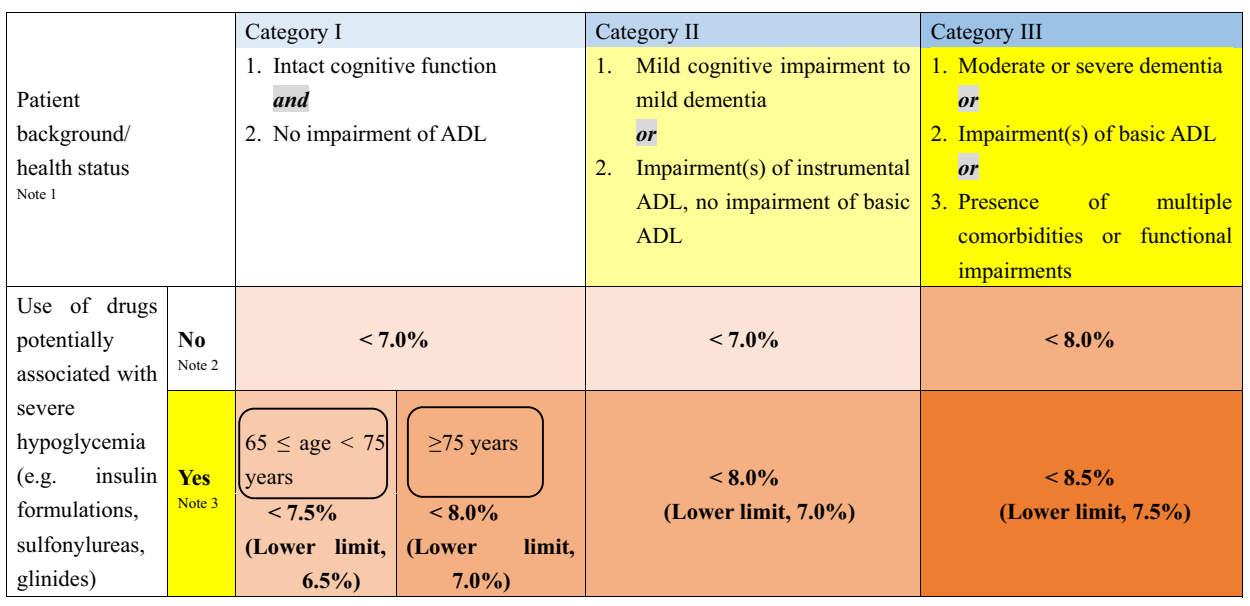

The glycemic target is to be determined for each patient by taking into account his/her age, duration of diabetes, risk for hypoglycemia, and any support available to the patient, as well as the patient's cognitive function, basic/instrumental ADL, and comorbidities/functional impairments, if elderly, while noting the potential risk of hypoglycemia that increases with age in each patient

$A D L$ activities of daily living; Note 1 Refer to the Japan Geriatrics Society website [9, 10], for the evaluation of the cognitive function, basic ADL (e.g. self-care abilities such as dressing, transferring, bathing, and toileting), and instrumental ADL (e.g. abilities to maintain an independent household such as shopping, meal preparation, taking medication, and handling finances,). In end-of-life care, priority is to be given to preventing significant hyperglycemia and subsequent dehydration and acute complications through appropriate therapeutic measures; Note 2 As in other age groups, the glycemic target is set at $<7.0 \%$ in the elderly for preventing diabetic complications. However, this could be set at $<6.0 \%$ for those thought likely to achieve glycemic control through diet and exercise therapy alone or those likely to achieve glycemic control with drug therapy without adverse reactions, or $8.0 \%$ for those in whom intensifying therapy may prove difficult. In either case, no lower limit is specified for the glycemic target. A glycemic target of $<8.5 \%$ may be allowed in patients thought to be in category III and therefore at risk of developing adverse reactions to multi-drug combination therapy or in those with serious comorbidities or poor social support; Note 3 In patients in whom priority should be given to preventing the onset/progression of diabetic complications due to their duration of disease, the glycemic target or its lower limit may be set for each elderly patient with appropriate measures in place to prevent severe hypoglycemia. Current treatments are to be continued in those less than 65 years of age despite their HbA1c values falling below their glycemic target or lower limit while on therapy, but care needs to be taken to monitor these patients for potential severe hypoglycemia. Glinides may be classified as drugs unlikely to be associated with severe hypoglycemia, as the onset of severe hypoglycemia varies depending on the type and amount of glinide used in a particular patient relative to the patient's glucose level

committee report in "Treatment Guide for Diabetes 2016-2017; 98" (Bunkodo, Tokyo, 2016) and "Practice Guideline for the Treatment for Diabetes in Japan 2016; 447-448" (Nankodo, Tokyo, Japan, 2016) in Japanese. This is the English version of that report and has been jointly published in Diabetology International (the official English journal of the JDS), Geriatrics and Gerontology International (the official English journal of the JGS), and Journal of Diabetes Investigation (the official journal of the Asian Association for the Study of Diabetes).

Members of the JDS/JGS Joint Committee on Improving Care for Elderly Patients with Diabetes. JDS: Masakazu Haneda (Head, Professor Emeritus, Visiting Professor, Asahikawa Medical University, Asahikawa, Japan), Nobuya Inagaki (Professor, Department of Diabetes, Endocrinology and Nutrition, Kyoto University Graduate
School of Medicine, Kyoto, Japan), Ryo Suzuki (Assistant Professor, Department of Diabetes and Metabolic Diseases, Graduate School of Medicine, The University of Tokyo, Tokyo, Japan), Hirotaka Watada (Professor, Department of Metabolism and Endocrinology, Juntendo University, Graduate School of Medicine, Tokyo, Japan). JGS: Hideki Ito (Head, C.E.O., Tokyo Metropolitan Hospital and Institute of Gerontology, Tokyo, Japan), Atsushi Araki (Executive Director, Department of Internal Medicine, Tokyo Metropolitan Hospital and Institute of Gerontology, Tokyo, Japan), Takashi Sakurai (Director, Center for Comprehensive Care and Research on Memory Disorders, National Center for Geriatrics and Gerontology, Aichi, Japan), Koutaro Yokote (Professor, Department of Medicine, Chiba University, Graduate School of Medicine, Chiba, Japan). 


\section{References}

1. National Health/Nutrition Survey 2012: Summary of survey results. Part 1: current status of diabetes. Ministry of Health, Labor and Welfare; 2012; p. 32-34 (in Japanese).

2. Huang ES, Laiteerapong N, Liu JY, et al. Rates of complications and mortality in older patients with diabetes mellitus: the diabetes and aging study. JAMA Intern Med. 2014;2(174):251-8.

3. Geller AI, Shehab N, Lovegrove MC, Kegler SR, Weidenbach KN, Ryan GJ, Budnitz DS. National estimates of insulin-related hypoglycemia and errors leading to emergency department visits and hospitalizations. JAMA Intern Med. 2014;174:678-86.

4. Whitmer RA, Karter AJ, Yaffe K, Quesenberry CP Jr, Selby JV. Hypoglycemic episodes and risk of dementia in older patients with type 2 diabetes mellitus. JAMA. 2009;301:1565-72.

5. Goto A, Arah OA, Goto M, Terauchi Y, Noda M. Severe hypoglycaemia and cardiovascular disease: systematic review and meta-analysis with bias analysis. BMJ. 2013;347:f4533. doi:10. 1136/bmj.f4533.

6. Araki A, Iimuro S, Sakurai T, Umegaki H, Iijima K, Nakano H, Oba K, Yokono K, Sone H, Yamada N, Ako J, Kozaki K, Miura H, Kashiwagi A, Kikkawa R, Yoshimura Y, Nakano T, Ohashi Y, Ito $\mathrm{H}$, the Japanese Elderly Diabetes Intervention Trial Study Group. Long-term multiple risk factor interventions in Japanese elderly diabetic patients: The Japanese Elderly Diabetes Intervention Trial (J-EDIT) - study design, baseline characteristics, and effects of intervention. Geriatr Gerontol. 2012;12(Suppl. 1):8-17.
7. Kirkman MS, Briscoe VJ, Clark N, Florez H, Haas LB, Halter JB, Huang ES, Korytkowski MT, Munshi MN, Odegard PS, Pratley RE, Swift CS. Diabetes in older adults. Diabetes Care. 2012;35:2650-64.

8. IDF Global Guideline. Managing older people with type 2 diabetes: Glucose control management and targets. 2013; 30-36.

9. Japanese Geriatrics Society. Methodology for evaluation of cognitive function and diagnosis of dementia-a useful tool for geriatric care (in Japanese). http://www.jpn-geriat-soc.or.jp/tool/ index.html.

10. Methodology for evaluation of ADL. Methodology for evaluation of cognitive function and diagnosis of dementia-a useful tool for geriatric care (in Japanese). http://www.jpn-geriat-soc.or.jp/tool/ index.html.

11. The Japan Geriatrics Society, Japan Agency for Medical Research and Development. Guidelines for Medical Treatment and its Safety in the Elderly 2015, Medical View Co., Ltd; Tokyo, 2015 (in Japanese).

12. Kojima T, Mizukami K, Tomita N, Arai H, Ohrui T, Eto M, Takeya Y, Isaka Y, Rakugi H, Sudo N, Arai H, Aoki H, Horie S, Ishii S, Iwasaki K, Takayama S, Suzuki Y, Matsui T, Mizukami F, Furuta K, Toba K, Akishita M; Working Group on Guidelines for Medical Treatment and its Safety in the Elderly. Screening Tool for Older Persons' Appropriate Prescriptions in Japanese: Report of the Japan Geriatrics Society Working Group on "Guidelines for Medical Treatment and its Safety in the Elderly". Geriatr Gerontol Int. 2016;16(9):983-1001. doi:10.1111/ggi. 12890 